\title{
Economic and ecological implications of geographic bias in pollinator ecology in the light of pollinator declines
}

\author{
C. R. Archer, C. W. W. Pirk, L. G. Carvalheiro and S. W. Nicolson \\ C. R. Archer (ruth.archer@zoology.up.ac.za), C. W. W. Pirk and S. W. Nicolson, Dept of Zoology and Entomology, Univ. of Pretoria, \\ Pretoria 0002, South Africa. - L. G. Carvalheiro, School of Biology, Univ. of Leeds, Leeds, LS29JT, UK, and Naturalis Biodiversity Center, \\ PO Box 9517, 2300RA, Leiden, the Netherlands.
}

\begin{abstract}
Understanding the causes and consequences of pollinator declines is a priority in ecological research. However, across much of the globe we have a poor understanding of pollinator assemblages, population trends and the ecological and economic importance of particular pollinators, due to a marked geographic bias in research effort. Here, we show that almost half the data cited in thirteen recent meta-analyses, which ask important and diverse questions in pollination ecology, were collected in just five countries: Australia, Brazil, Germany, Spain and the USA. In contrast, the entire continent of Africa contributed only $4 \%$ of the data. We believe that the consequences of this geographic bias are severe. Foremost, pollinator assemblages (and possibly their sensitivity to ecological drivers) can greatly vary among these regions. In addition, many communities that rely on pollinators, bees in particular, for food security and wealth generation are in geographic regions where our understanding of pollination is poor. Collecting accurate information on pollinator populations in data deficient areas will allow us to identify vulnerable populations and species and so better target conservation measures. Moreover, it will help us to determine if our current understanding of pollinator losses, based on data collected in a few locations and on the species that predominate in those regions, is representative of the wide diversity of ecosystems. We propose means of collecting such data given socioeconomic constraints.
\end{abstract}

An estimated $87.5 \%$ of the world's wild plants are pollinated by animals (Ollerton et al. 2011). Pollinators are therefore vital to preserving high agricultural productivity (Klein et al. 2007) and ecosystem function (Cox and Elmqvist 2000). However, wild insect pollinators including moths (Fox 2012), butterflies (Maes and Van Dyck 2001) and bees (Biesmeijer et al. 2006, Kosior et al. 2007, Cameron et al. 2011) have declined in both abundance and diversity, while, at least in some parts of the world, managed populations of honeybees Apis mellifera have recently experienced large scale, sudden colony losses (van Engelsdorp et al. 2008). Understanding the causes of these declines is an absolute priority in ecological research.

Preventing pollinator declines and preserving food security requires basic research to characterise the pollination biology of different crops, important pollinator species and the impact of pollinator deficits on plant productivity (Klein et al. 2007, Bartomeus and Winfree 2013). Additionally, targeted efforts must be made to identify the rates and drivers of pollinator loss internationally (Potts et al. 2010). Indeed, one response to widespread pollinator losses has been an increase in the number of studies on pollinators and pollination (Viana et al. 2012). Meta-analyses have integrated these data to better understand the causes and consequences of pollinator losses (Ricketts et al. 2008,
Garibaldi et al. 2011, Kennedy et al. 2013). However, a marked geographic bias in research effort in pollinator research (Mayer et al. 2011, Bartomeus et al. 2013) means that we are unsure if patterns reported in such meta-analyses are representative of pollinator ecology across a wide diversity of ecosystems.

We aim to show that geographic bias in research intensity means that there are large gaps in our understanding of pollinator ecology and, more specifically, pollinator losses. We then wish to discuss the economic and ecological consequences of this geographic bias, with reference to losses of bees and other pollinators. Finally, we propose ways of filling geographic gaps in our understanding, principally in less economically developed countries, where logistical constraints make research more challenging.

\section{Pollination ecology and pollinator loss - where are the gaps?}

A recent attempt to identify key questions in pollination ecology for the 21 st century highlighted the fact that, outside of Europe and North America, our understanding of pollinator ecology and diversity is poor (Mayer et al. 2011). Indeed, Viana et al. (2012) showed that our understanding of the effects of landscape structure on pollination is based 
primarily on data collected in temperate rather than tropical ecosystems and, amongst these temperate zones, most data published since the turn of the century comes from Germany, the UK and the USA. Such bias is also likely to characterise pollinator loss research because across much of the globe we have little or no data monitoring pollinator assemblages or population trends (Goulson et al. 2008, Winfree 2010). Indeed, Winfree et al. (2011) detected geographic bias in the published literature exploring how native pollinators respond to land use changes, finding that studies conducted in Europe or North America account for 52\% of all recorded responses.

We wished to determine if the geographic bias highlighted by Viana et al. (2012) and Winfree et al. (2011) represents a general trend in meta-analyses exploring pollinator ecology and more specifically, pollinator losses. We focus on metaanalyses as these collate large bodies of literature to extract general patterns, which may have a strong influence on policy makers and conservation managers. We assume that the frequency with which a country contributes data to such analyses reflects either the research intensity in that country or the utility or accessibility of data collected there. We do not wish to conduct formal quantitative analyses on these data but merely to show how well attempts to synthesise primary literature using meta-analytical techniques reflect global research on plant-pollinator interactions.

To this end, we searched for meta-analyses relating to pollinator ecology and loss published since 2004, using the search terms Topic $=$ (quantitative SAME synthesis OR synthesize SAME studies OR synthesize SAME literature
OR synthesize SAME data OR meta-analysis OR Web SAME knowledge OR Googlescholar) AND Title $=($ bees OR pollinat* OR animal mutualist OR plant SAME reproduc*) in Web of Knowledge. We supplemented this collection with haphazard searches using the key terms listed above in Google Scholar, to detect any missing papers. We excluded any meta-analyses based on laboratory studies or those that focused on a specific region, pollinator species, or a driver of pollinator loss that may be specific to a geographic location, for example, a particular pesticide. Where meta-analyses used the same dataset (Vamosi et al. 2006, Alonso et al. 2010), we selected the more recent publication for analyses. We then determined where the data were collected for all the studies cited by these analyses (Table 1). We excluded any unpublished datasets because we wished to independently confirm the geographic origins of data cited rather than relying on information contained within supplementary files accompanying meta-analyses online.

This literature search yielded 13 meta-analyses, of which five focused generally on pollinator ecology (Vázquez et al. 2005, Burkle et al. 2007, Alonso et al. 2010, Ollerton et al. 2011, Romero et al. 2011), five on how pollinators or pollination services are responding to disturbances (Aguilar et al. 2006, Ricketts et al. 2008, Morales and Traveset 2009, Garibaldi et al. 2011, Ferreira et al. 2013.) and three focused specifically on bees (Winfree et al. 2009, Williams et al. 2010, Kennedy et al. 2013). These analyses cited 652 papers, of which 101 were duplicated across different metaanalyses. Eleven of those papers used data from more than one country. If these data were collected within the same

Table 1. The percentage of published data cited by each meta-analysis from each geographic region, rounded to the nearest whole value. Note - Oceania includes all data from Australia, New Zealand, Melanesia, Micronesia and Polynesia. For Vázquez et al. (2005), papers were taken from the 'pollination' rather than the 'seed dispersal' data set.

\begin{tabular}{|c|c|c|c|c|c|c|}
\hline \multirow[b]{2}{*}{ Study } & \multicolumn{6}{|c|}{ Continent } \\
\hline & Africa & Asia & Europe & N. America & Oceania & S. America \\
\hline \multicolumn{7}{|l|}{ Pollinator ecology } \\
\hline \multicolumn{7}{|l|}{ Animal pollination services } \\
\hline Alonso et al. 2010 & 3 & 12 & 22 & 45 & 8 & 10 \\
\hline Burkle et al. 2007 & 0 & 4 & 19 & 74 & 0 & 4 \\
\hline Ollerton et al. 2011 & 0 & 13 & 26 & 30 & 4 & 26 \\
\hline Vázquez et al. 2005 & 0 & 5 & 24 & 67 & 0 & 5 \\
\hline \multicolumn{7}{|l|}{ Pollinator behaviour } \\
\hline Romero et al. 2011 & 8 & 12 & 12 & 62 & 0 & 8 \\
\hline Total excluding duplicates & 3 & 11 & 22 & 49 & 6 & 10 \\
\hline \multicolumn{7}{|c|}{ Effects of disturbances on pollinators or pollination systems } \\
\hline \multicolumn{7}{|c|}{ Landscape effects on pollination services, plant-pollinator interactions and plant reproductive success } \\
\hline Aguilar et al. 2006 & 9 & 7 & 30 & 27 & 14 & 13 \\
\hline Ferreira et al. 2013 & 3 & 14 & 28 & 32 & 4 & 19 \\
\hline Garibaldi et al. 2011 & 8 & 13 & 4 & 46 & 17 & 13 \\
\hline Ricketts et al. 2008 & 5 & 11 & 0 & 53 & 21 & 11 \\
\hline \multicolumn{7}{|c|}{ Impact of alien versus native plants on pollinator visitation and plant reproductive success } \\
\hline Morales and Traveset 2009 & 6 & 8 & 31 & 50 & 0 & 6 \\
\hline \multicolumn{7}{|c|}{ Local and landscape effects on wild bee pollinators in agricultural systems } \\
\hline Kennedy et al. 2013 & 7 & 7 & 15 & 52 & 7 & 11 \\
\hline \multicolumn{7}{|l|}{ Impact of anthropogenic disturbances on bees } \\
\hline Winfree et al. 2009 & 2 & 17 & 31 & 29 & 4 & 17 \\
\hline Williams et al. 2010 & 5 & 26 & 26 & 26 & 5 & 11 \\
\hline Total excluding duplicates & 5 & 11 & 29 & 32 & 7 & 16 \\
\hline Grand total all papers, excluding duplicates & 4 & 11 & 25 & 41 & 6 & 13 \\
\hline
\end{tabular}



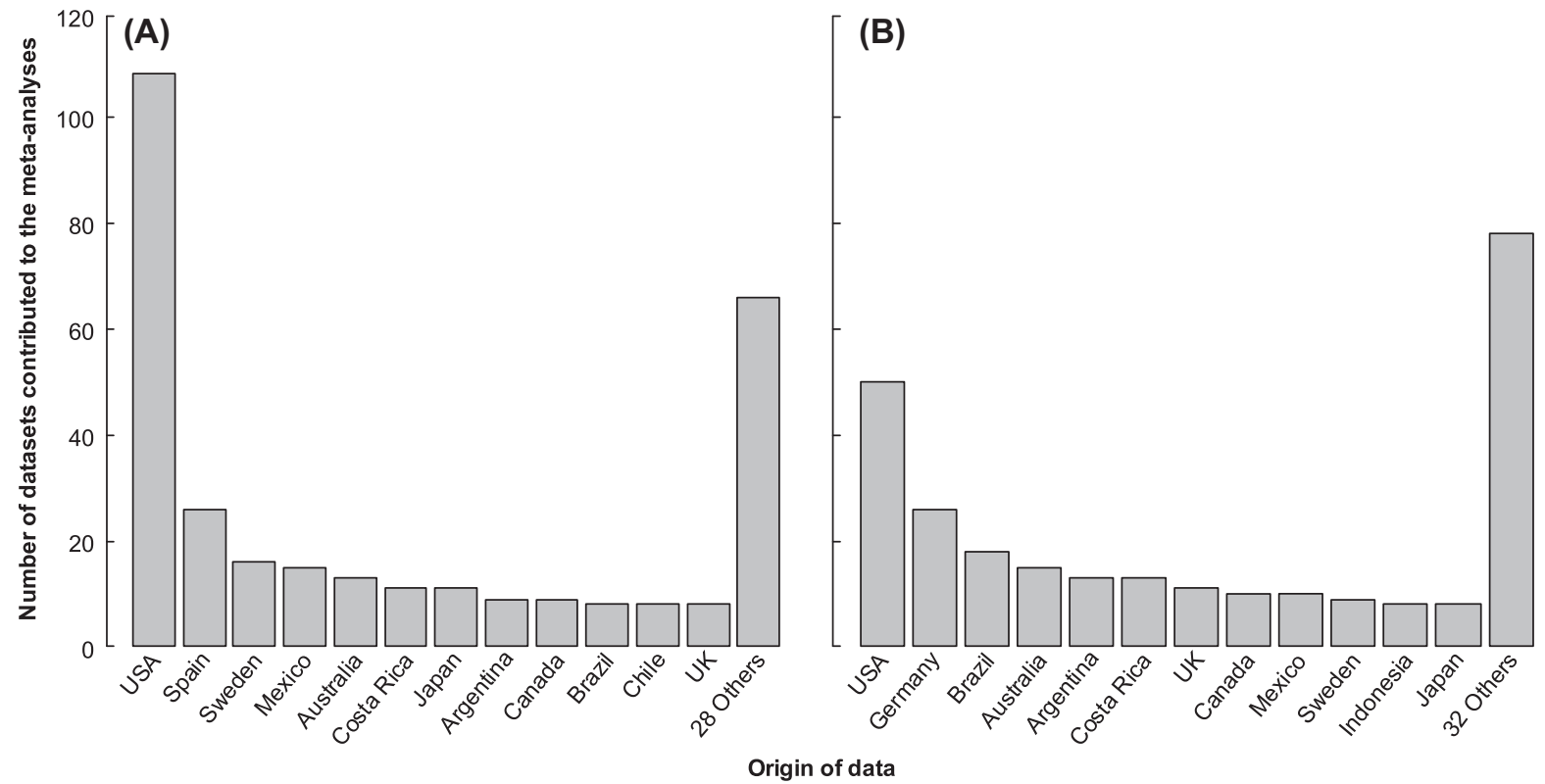

Figure 1. Countries where the data cited by the 13 meta-analyses (Table 1), excluding duplicate references, were collected for either studies asking general questions in pollinator ecology (A) or studies asking how pollinators or pollination systems are affected by perturbation (B).

geographic region, they were only counted once in summaries of wider regional trends.

Irrespective of whether these papers focused on general pollinator ecology, or how pollination systems or pollinators respond to environmental changes, the results were largely consistent (Fig. 1A-B, Table 1). The majority of the data cited in these analyses was collected in either Europe or North America. More specifically, just five countries, USA, Spain, Australia, Germany and Brazil contributed nearly half $(47 \%)$ of all data cited in these meta-analyses.

Basic pollinator ecology research relied more heavily on data from the United States (35\%) than studies asking how pollination systems or pollinators respond to perturbation, where the USA contributed $18 \%$ of data. Amongst the three analyses focusing on bees, Winfree and colleagues (2009) drew largely on data collected in Germany (21\%) and the United States (19\%), while in Kennedy et al. (2013), 29\% of data cited came from the USA alone. More balance was evident in Williams et al. (2010), where Europe, North America and Asia each contributed $26 \%$ of data to the dataset. Evidently, our understanding of pollination ecology in general and, more specifically the drivers of pollinator declines, is based on data collected from Europe and North America. Accordingly, with the exception of some social bees, we do not know the extent to which many pollinator taxa are declining or understand geographic variation in pollinator loss (Mayer et al. 2011).

\section{The crucial importance of filling in knowledge gaps}

We believe that to prevent and understand pollinator loss we must expand the range of pollinator ecology research and its accessibility to researchers worldwide. One reason for this is that there is substantial geographic variation in economic vulnerability to pollinator loss (Gallai et al. 2009) (Table 2) and although some areas that are most vulnerable are well studied, e.g. regions of the United States, this is not true across all geographic regions. For example, west Africa and central Asia are approximately as economically vulnerable to

Table 2. The economic vulnerability to pollinator loss, as calculated by Gallai and colleagues (2009), is the ratio of the economic value of insect pollinators of the 100 most important commodity crops for human consumption to the total economic value of those crops. As in Gallai et al. (2009), regions with high vulnerability to pollinator loss $(\geq 10 \%)$ are highlighted in bold. The number of papers refers to data-sets used in the meta-analyses, after duplicates were removed, from each geographic region.

\begin{tabular}{|c|c|c|}
\hline $\begin{array}{l}\text { Geographical region and } \\
\text { sub-region (following FAO, } \\
<\text { www.fao.org }>\text { ) }\end{array}$ & $\begin{array}{l}\text { Vulnerability } \\
\text { of region }\end{array}$ & $\begin{array}{c}\text { Number of } \\
\text { papers } \\
\text { contributed to } \\
\text { meta-analyses }\end{array}$ \\
\hline
\end{tabular}

Africa

$\begin{array}{lll}\text { Central Africa } & 7 & 1\end{array}$

East Africa

North Africa

South Africa

West Africa

$5 \quad 7$

Asia

Central Asia

East Asia

Middle East Asia

Oceania

South Asia

South East Asia

Europe

European Union (25 members)

Non EU25

11

7

$6 \quad 12$

North America

Bermuda, Canada and USA

South and Central America

Central America and Caribbean

South America
10 
pollinator loss as North America (Gallai et al. 2009), but these geographic regions did not contribute a single paper to the meta-analyses referenced in the previous section. Accordingly, crops grown in these regions are often severely understudied (Rodger et al. 2004). To protect agricultural activities in these highly vulnerable but data poor regions requires research to identify important pollinators in these areas and the threats facing them and to devise strategies to reduce these threats or buffer any losses e.g. by identifying alternative pollinators.

However, Gallai et al. (2009) relied on the market value of insect pollinated crops to calculate economic vulnerability to pollinator declines and so overlooked the contribution of pollinators to subsistence farming or as a food source for households. In less economically developed countries (LEDCs) these latter measures may be more relevant than market value of crops in calculating a region's economic vulnerability to pollinator loss; hunter gatherer communities across Africa and Asia rely on wild honeybee colonies for food (summarised in Venkataraman et al. 2013), while small scale apiculture generates vital income and provides food security for farmers in some of the world's poorest communities. For example, in Kenya (Sande et al. 2009), Zimbabwe (Chazovachii et al. 2012) and Nepal (Pokhrel 2009) beekeeping is used as a means of generating income and promoting food security. In communities such as these, bee declines could rapidly and severely impact on local food security. Research aimed at preventing the decline of pollinators in such regions where the socioeconomic consequences of pollinator loss could be severe should be a priority (Rodger et al. 2004).

Redirecting the focus of pollinator loss research is also important from an ecological perspective, both in terms of preventing and understanding declines. To discuss why this is, by necessity, we focus on bees, which are important pollination services providers (Klein et al. 2007) and are the most heavily studied pollinator guild (Potts et al. 2010). Foremost, collecting accurate information on pollinator populations and the threats facing them is necessary so that we may identify and conserve vulnerable pollinator lineages (Williams and Osborne 2009): these include monotypic genera endemic to understudied regions, such as Calloprosopis bees in Kenya (Eardley et al. 2010). Such species are taxonomically important and therefore targets for conservation irrespective of their efficiency as pollinators (Winfree 2010). By expanding the range of pollinator research we may also gain a better understanding of the drivers of pollinator losses in regions where such declines are already a cause of great concern, as in Europe or the USA. For example, we know that several factors contribute towards bee species declines including habitat destruction, agricultural intensification, pests and pathogens (Potts et al. 2010, Vanbergen and the Insect Pollinators Initiative 2013). Negative impacts are often increased in bees exposed to two or more of these stressors simultaneously. For example, sub-lethal doses of the pesticides fipronil and thiacloprid reduce survival in honeybees previously infected by the parasite Nosema ceranae (Vidau et al. 2011). Unravelling how different factors interact to drive bee species declines is a primary aim of pollinator loss research (Potts et al. 2010) but in Europe and North America this is challenging, particularly on a landscape scale. This is because these regions are characterised by high levels of anthropogenic disturbance and so bees are exposed to a multitude of stressors simultaneously (i.e. agricultural intensification, pesticide use, habitat destruction). In other regions of the world, where bee populations are not exposed to this full suite of stressors, it may be easier to disentangle the effects of different drivers of decline. For example, while high levels of urbanisation (Antrop 2004) and agricultural intensification (Stoate et al. 2001) in Europe mean that there is little natural habitat, Africa presents a very different situation (Moritz et al. 2007). In honeybees, the ectoparasite Varroa, which can play a major part in causing winter losses of colonies (Guzmán-Novoa et al. 2010), is widespread across hives in Europe but absent (to date) from Australia (Rinderer et al. 2013). By studying populations that are not exposed to the full range of stressors that affect European and North American pollinators, we may be able to better understand the role of individual drivers of pollinator declines and interactions between them.

Finally, geographic skew in research effort inevitably promotes taxonomic bias towards species found in particular regions. In the case of bees, that may help explain why most research on pollinator declines has been carried out on the genus Bombus (discussed in Bartomeus et al. 2013). However, there are nearly 20000 species of bees (Pickering and Ascher 2012), Bombus species being only a minority, and each of these may respond differently to anthropogenic disturbances. Indeed, Bombus species appear to be more vulnerable to drivers of change than other bee species in Europe (Carvalheiro et al. 2013) and the USA (Bartomeus et al. 2013). The reason for such differences may be that species differ in a suite of traits that affect vulnerability to particular stressors or environmental changes. For example, nest location (below or above the ground) affects how bees tolerate habitat loss (Williams et al. 2010). Likewise, solitary bees are less affected by isolation from natural habitats and exposure to pesticides than social bees (Williams et al. 2010). On a finer scale, even variation within a single species can affect how bees respond to particular disturbance. For example, the 24 subspecies of honeybee (Ruttner 1988) can show behavioural (Breed et al. 2004) and physiological (Brillet et al. 2002) differences, which can influence responses to particular stressors. If we compare African and European populations (i.e. from the only continents where Apis mellifera is endemic), the ectoparasite Varroa can cause overwinter losses of colonies of Apis mellifera of European origin (Guzmán-Novoa et al. 2010) but has little or no effect on African honeybees (Strauss et al. 2013). These pronounced differences between bee (sub)species limit how far we can extrapolate from our existing understanding of bee declines, which is built on a handful of species. Although many of the most severe challenges facing bees may be consistent throughout the range of a species (e.g. habitat destruction and agricultural intensification) (Vanbergen and the Insect Pollinators Initiative 2013), the impacts of many drivers of bee declines are likely to differ across (sub)species and geographic regions and so conservation methods must be tailored accordingly (Dietemann et al. 2009, Human et al. 2011). 


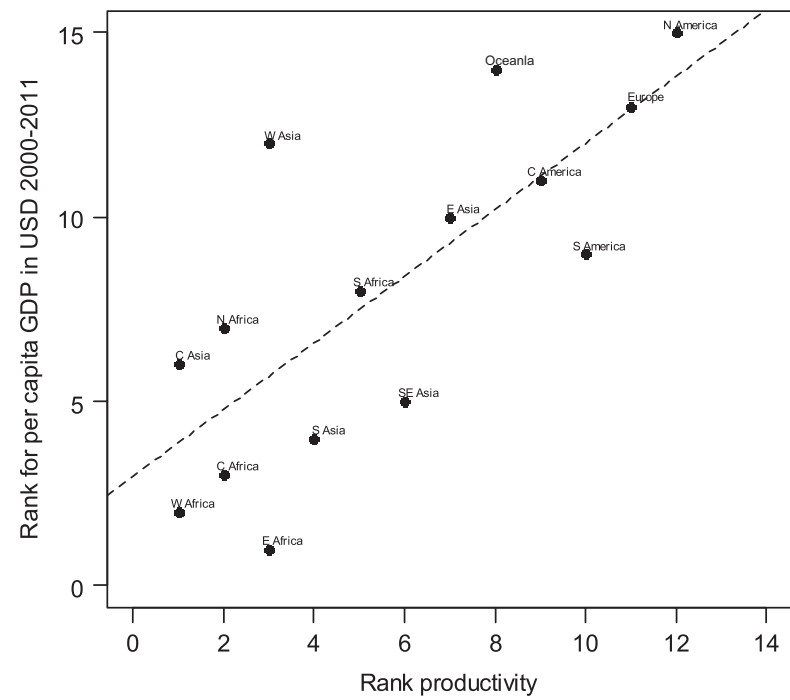

Figure 2. The number of data-sets cited in the 13 meta-analyses, excluding duplicates, ranked from lowest to highest from each geographic region plotted against the ranking of that region according to average per capita GDP from 2000-2011 (taken from the United Nations Statistics Division: <http://unstats.un.org >). Abbreviations $-\mathrm{E}=$ eastern, $\mathrm{C}=$ central, $\mathrm{N}=$ northern, $\mathrm{S}=$ southern and $\mathrm{W}=$ western. Analysis with a generalized linear model and quasipoisson error structure in $\mathrm{R}$ ver. 3.0.0 and backwards model selection shows that GDP has a significant positive effect on productivity $\left(\mathrm{F}_{14,13}=15.777, \mathrm{p}=0.002\right)$.

\section{Strategies for filling in the missing pieces of the pollinator loss puzzle}

Geographic gaps are a pervasive problem in ecological research (Martin et al. 2012); in fields as diverse as invasion biology (Pyšek et al. 2008) and tropical ecology (Stocks et al. 2008), LEDCs are understudied. Although the aim of this article was not to comprehensively review the causes of geographic bias in ecology research, as this has been done elsewhere, simply plotting the number of papers that each geographic region contributed to the meta-analyses discussed previously against that region's per capita GDP shows a clear trend; more affluent regions contributed more papers to the meta-analyses (Fig. 2). This means that LEDCs that lack the research community, institutions, established field stations or infrastructure to facilitate research are often understudied and their ecology poorly understood (Pyšek et al. 2008, Stocks et al. 2008, Martin et al. 2012).

While the scarcity of papers from certain regions in the meta-analyses reviewed here almost certainly reflects a lack of data, there is likely a sizeable pool of data for many countries which is simply inaccessible or not amenable to inclusion in a meta-analysis. Indeed, pollinator research frequently cites unpublished data-sets (Ricketts et al. 2008, Morales and Traveset 2009, Winfree et al. 2009). Likewise, data collected by commercial organisations providing pollination services are seldom included in scientific journals but instead in lay agricultural publications (Rodger et al. 2004), which are obviously not indexed by scientific search engines. Even where data are in the public domain a lack of standardised procedures and protocols across studies (Westphal et al. 2008) limits how readily data can be incorporated into meta-analyses. Hence, although geographic bias in pollinator ecology undoubtedly reflects research intensity, data being inaccessible and a lack of standardised protocols almost certainly exacerbates the problem.

Given that many understudied regions have poor infrastructure or funding opportunities, how can we, firstly, collect data from these regions and, secondly, ensure data are both useable and accessible? Foremost, research in understudied regions should be facilitated by international collaborations (Viana et al. 2012). Collaborative efforts could also be used to identify regions at high risk of pollinator declines. High risk areas could be defined as those that are data poor, economically most reliant on insect pollinators and faced with emerging threats to pollinator health, with emphasis given to those drivers of decline that are 'general' i.e. known to affect a range of pollinator guilds across varying habitats. These data could be incorporated alongside our understanding of how life-history or morphological traits affect vulnerability to particular stressors (Williams et al. 2010), and collated online, with research, funding or practical information on how to mitigate pollinator losses targeted to particularly vulnerable regions.

Such collaborative groups should help develop and implement standardised, repeatable protocols that can be applied internationally (Westphal et al. 2008). For example, the International Pollinators Initiative, a collaboration between researchers from seven countries (Brazil, Ghana, India, Kenya, Nepal, Pakistan and South Africa) has devised a protocol for assessing pollination deficits, designed with LEDCs in mind (Vaissière et al. 2011). Such protocols facilitate the rapid collection of data that can readily be compared across studies separated both temporally and spatially (Westphal et al. 2008). A protocol listing baseline data that studies of pollination should collect, such as land use information, a range of climatic variables and floral diversity and abundance would be equally useful.

On a local scale, we also need to identify existing networks that can help us gather data in areas with poor infrastructure. Some tremendously useful information regarding bee species abundance has been collected by members of the public in the UK (Lye et al. 2012) and, although a poor infrastructure and little access to the internet has typically made citizen science challenging in LEDCs, this is changing. Citizens, equipped with mobile phones with cameras, GPS and internet capabilities are helping update distribution records of mammal species in South Africa (mammalMAP) and data are being uploaded to an online repository. The same technology could be applied to monitor pollinators, particularly in areas where pollinator species taxonomy is well understood. Additionally, in LEDCs, both governments and NGOs are organising groups of small scale beekeepers, which need advice on how to recognise and prevent honeybee diseases (Woldewahid et al. 2012). This provides an opportunity for information transfer where researchers promote standardised, simple means of assaying colony health and in turn may collect data on bee diseases in remote rural communities.

Efforts should also be made to collate data that are not readily accessible. For example, museum specimens, which 
may be used to study temporal trends in pollinator distributions and community level trends (Bartomeus et al. 2013, Carvalheiro et al. 2013), could be digitised and incorporated with data collected via citizen science, to create a virtual museum accessible by researchers internationally. Such a repository could also be used to store data cited in research papers or theses but not destined for publication, as well as records of university insect specimens.

It will certainly be challenging to devise means to collect data where currently we have very little; poor infrastructure, small research communities and limited funding opportunities are sizeable barriers. We need to think of ways to breach these. In the interim, we must recognise that there is a careful compromise to be made between using our current (skewed) understanding of pollinator losses to guide and inform conservation efforts elsewhere and recognising that variation between species, habitats and geographical regions limits how far we may extrapolate from these data.

Acknowledgements - This work was funded jointly by a grant from the BBSRC, NERC, the Wellcome Trust, Defra, and the Scottish Government under the Insect Pollinators Initiative (BB/I000968/1). We also wish to acknowledge the support of the National Research Foundation of South Africa and the University of Pretoria. LGC is funded by the EU FP7 projects 'Status and Trends of European Pollinators' (244 090, < www.STEP-project.net >).

\section{References}

Aguilar, R. et al. 2006. Plant reproductive susceptibility to habitat fragmentation: review and synthesis through a meta-analysis. - Ecol. Lett. 9: 968-980.

Alonso, C. et al. 2010. Is reproduction of endemic plant species particularly pollen limited in biodiversity hotspots? - Oikos 119: 1192-1200.

Antrop, M. 2004. Landscape change and the urbanization process in Europe. - Landscape Urban Planning 67: 9-26.

Bartomeus, I. and Winfree, R. 2013. Pollinator declines: reconciling scales and implications for ecosystem services. - F1000 Res. 2:146.

Bartomeus, I. et al. 2013. Historical changes in northeastern US bee pollinators related to shared ecological traits. - Proc. Natl Acad. Sci. USA 110: 4656-4660.

Biesmeijer, J. C. et al. 2006. Parallel declines in pollinators and insect-pollinated plants in Britain and the Netherlands. - Science 313: 351-354.

Breed, M. D. et al. 2004 Defensive behavior of honey bees: organization, genetics and comparisons with other bees. - Annu. Rev. Entomol. 49: 271-298.

Brillet, C. et al. 2002. Racial differences in division of labor in colonies of the honey bee (Apis mellifera). - Ethology 108: $115-126$

Burkle, L. A. et al. 2007. Predicting the effects of nectar robbing on plant reproduction: implications of pollen limitation and plant mating system. - Am. J. Bot. 94: 1935-1943.

Cameron, S. A. et al. 2011. Patterns of widespread decline in North American bumble bees. - Proc. Natl Acad. Sci. USA 108: 662-667.

Carvalheiro, L. G. et al. 2013. Species richness declines and biotic homogenisation have slowed down for NW-European pollinators and plants. - Ecol. Lett. 16: 870-878.
Chazovachii, B. et al. 2012. Livelihood resilient strategies through beekeeping in Chitanga village, Mwenezi District, Zimbabwe. - Sustain. Agric. Res. 2: 124-132.

Cox, P. A. and Elmqvist, T. 2000. Pollinator extinction in the Pacific Islands. - Conserv. Biol. 14: 1237-1239.

Dietemann, V. et al. 2009. Is there a need for conservation of honeybees in Africa? - Apidologie 40: 285-295.

Eardley, C. et al. 2010. The bee genera and subgenera of sub-Saharan Africa. - Koninklijk Belgisch Instituut voor Natuurwetenschappen.

Ferreira, P. A. et al. 2013. What do we know about the effects of landscape changes on plant-pollinator interaction networks? - Ecol. Ind. 31: 35-40.

Fox, R. 2012. The decline of moths in Great Britain: a review of possible causes. - Insect Conserv. Divers. 6: 5-19.

Gallai, N. et al. 2009 Economic valuation of the vulnerability of world agriculture confronted with pollinator decline. - Ecol. Econom. 68: 810-821.

Garibaldi, L. A. et al. 2011. Stability of pollination services decreases with isolation from natural areas despite honey bee visits. - Ecol. Lett. 14: 1062-1072.

Goulson, D. et al. 2008. Decline and conservation of bumble bees. - Annu. Rev. Entomol. 53: 191-208.

Guzmán-Novoa, E. et al. 2010. Varroa destructor is the main culprit for the death and reduced populations of overwintered honey bee (Apis mellifera) Colonies in Ontario, Canada. - Apidologie 41: 443-450.

Human, H. et al. 2011. The honeybee disease American foulbrood - an African perspective. - Afr. Entomol. 19: 551-557.

Kennedy, C. M. et al. 2013. A global quantitative synthesis of local and landscape effects on wild bee pollinators in agroecosystems. - Ecol. Lett. 16: 584-599.

Klein, A-M. et al. 2007. Importance of pollinators in changing landscapes for world crops. - Proc. R. Soc. B. 274: 303-313.

Kosior, A. et al. 2007. The decline of the bumble bees and cuckoo bees (Hymenoptera: Apidae: Bombini) of western and central Europe. - Oryx 41: 79-88.

Lye, G. C. et al. 2012. Using citizen science to monitor Bombus populations in the UK: nesting ecology and relative abundance in the urban environment. - J. Insect Conserv. 16: 697-707.

Maes, D. and Van Dyck, H. 2001. Butterfly diversity loss in Flanders (north Belgium): Europe's worst case scenario? - Biol. Conserv. 99: 263-276.

Martin, L. J. et al. 2012. Mapping where ecologists work: biases in the global distribution of terrestrial ecological observations. - Front. Ecol. Environ. 10: 195-201.

Mayer, C. et al. 2011. Pollination ecology in the 21st century: key questions for future research. - J. Pollination Ecol. 3: 8-23.

Morales, C. L. and Traveset, A. 2009. A meta-analysis of impacts of alien vs. native plants on pollinator visitation and reproductive success of co-flowering native plants. - Ecol. Lett. 12: 716-728.

Moritz, R. et al. 2007. The size of wild honeybee populations (Apis mellifera) and its implications for the conservation of honeybees. - J. Insect Conserv. 11: 391-397.

Ollerton, J. et al. 2011. How many flowering plants are pollinated by animals? - Oikos 120: 321-326.

Pickering, J. and Ascher, J. 2012. World bee diversity. Discover life. $-<$ www.discoverlife.org $>$.

Pokhrel, S. 2009. Comparitive benefits of beekeeping enterprise in Chitwan, Nepal. - J. Agric. Environ. 10: 46-59.

Potts, S.G. et al. 2010. Global pollinator declines: trends, impacts and drivers. - Trends Ecol. Evol 25: 345-353.

Pyšek, P. et al. 2008. Geographical and taxonomic biases in invasion ecology. - Trends Ecol. Evol. 23: 237-244.

Ricketts, T. H. et al. 2008. Landscape effects on crop pollination services: are there general patterns? - Ecol. Lett. 11: 499-515. 
Rinderer, T. E. et al. 2013. Responses to Varroa destructor and Nosema ceranae by several commercial strains of Australian and North American honeybees (Hymenoptera: Apidae). - Aust. J. Entomol. 52: 156-163.

Rodger, J. G. et al. 2004. African pollination studies: where are the gaps. - Int. J. Trop. Insect Sci. 24: 5-28.

Romero, G. Q. et al. 2011. A meta-analysis of predation risk effects on pollinator behaviour. - PloS ONE 6: e20689.

Ruttner, F. 1988. Biogeography and taxonomy of honeybees. - Springer.

Sande, S. O. et al. 2009. Proximity to a forest leads to higher honey yield: another reason to conserve. - Biol. Conserv. 142: 2703-2709.

Stoate, C. et al. 2001. Ecological impacts of arable intensification in Europe. - J. Environ. Manage. 63: 337-365.

Stocks, G. et al. 2008. The geographical and institutional distribution of ecological research in the tropics. - Biotropica 40: 397-404.

Strauss, U. et al. 2013. Seasonal prevalence of pathogens and parasites in the savannah honeybee (Apis mellifera scutellata). - J. Invertr. Pathol. 114: 45-52.

Vaissière, B. E. et al. 2011. Protocol to detect and assess pollination deficits in crops: a handbook for its use. - FAO, United Nations.

Vamosi, J. C. et al. 2006. Pollination decays in biodiversity hotspots. - Proc. Natl Acad. Sci. USA 103: 956-961.

Vanbergen, A. J. and the Insect Pollinators Initiative. 2013. Threats to an ecosystem service: pressures on pollinators. - Front. Ecol. Environ. 11: 251-259.

van Engelsdorp, D. et al. 2008. A survey of honey bee colony losses in the U.S., fall 2007 to spring 2008. - PLoS ONE 3: e4071.
Vázquez, D. P. et al. 2005. Interaction frequency as a surrogate for the total effect of animal mutualists on plants. - Ecol. Lett. 8: 1088-1094.

Venkataraman, V. V. et al. 2013. Tree climbing and human evolution. - Proc. Natl Acad. Sci. USA 110: 1237-1242.

Viana, B. F. et al. 2012. How well do we understand landscape effects on pollinators and pollination services? - J. Pollination Ecol. 7: 31-41.

Vidau, C. et al. 2011 Exposure to sublethal doses of fipronil and thiacloprid highly increases mortality of honeybees previously infected by Nosema ceranae. - PLoS ONE 6: e21550.

Westphal, C. et al. 2008. Measuring bee diversity in different European habitats and biogeographical regions. - Ecol. Monogr. 78: 653-671.

Williams, P. H. and Osborne. J. L. 2009. Bumblebee vulnerability and conservation world-wide. - Apidologie 40: 367-387.

Williams, N. M. et al. 2010. Ecological and life-history traits predict bee species responses to environmental disturbances. - Biol. Conserv. 143: 2280-2291.

Winfree, R. 2010. The conservation and restoration of wild bees. - Ann. N. Y. Acad. Sci. 1195: 169-197.

Winfree, R. et al. 2009. A meta-analysis of bees' responses to anthropogenic disturbance. - Ecology 90: 2068-2076.

Winfree, R. et al. 2011. Native pollinators in anthropogenic habitats. - Annu. Rev. Ecol. Evol. Syst. 42: 1-22.

Woldewahid, G. et al. 2012. Market-oriented beekeeping development to improve smallholder income: results of development experiences in Atsbi-Womberta district, northern Ethiopia. - IPMS Case Study. Nairobi: Kenya: ILRI. 